\title{
O Software GeoGebra no Ensino da Matemática: Relatos a partir de Teses e Dissertações
}

\author{
João Carlos Alves Pereira Junior ${ }^{1}$, Daniel Bonadiman Bertol ${ }^{2}$ \\ ${ }^{1}$ Secretaria de Estado de Educação do Paraná - SEED/PR \\ ${ }^{2}$ Instituto Federal do Paraná - IFPR \\ joaocarlos14jr@gmail.com, danielbertol61@gmail.com
}

\begin{abstract}
This article aims to map and investigate which discussions have been privileged in investigations involving GeoGebra software that has been outstanding in the Mathematics Education scenario. We analyzed doctoral dissertations and masters dissertations (academic and professional) available in the CAPES Online Catalog of Theses and Dissertations in 2017 and 2018. To select the analyzed works, the word "GeoGebra" was used as a keyword, refining the search for area of concentration in Mathematics Education. This is an interpretative analysis that sought to identify in the titles and abstracts of the works the levels of education, the subjects of the research, the mathematical contents addressed and the mode of investigation.
\end{abstract}

Resumo. Este artigo tem por objetivo mapear e investigar quais discussões tem sido privilegiadas nas investigações envolvendo o software GeoGebra que tem se destacado no cenário da Educação Matemática. Analisamos teses de doutorado e dissertações de mestrados (acadêmico e profissional) disponíveis no Catálogo online de Teses e Dissertações da CAPES no período de 2017 e 2018. Para seleção dos trabalhos analisados foi utilizada a palavra "GeoGebra" como palavra-chave, refinando a busca para área de concentração em Educação Matemática. Trata-se de uma análise interpretativa que buscou identificar nos títulos e resumos dos trabalhos os níveis de ensino, os sujeitos das pesquisas, os conteúdos matemáticos abordados e a modalidade de investigação.

\section{Introdução}

As tecnologias digitais têm favorecido o ensino e a aprendizagem de Matemática [Estevam et al., 2018], porque auxiliam a experimentação, possibilitando a visualização e simulação de objetos matemáticos. Um dos softwares que tem se destacado no cenário da Educação Matemática é o GeoGebra por se tratar de um software livre, que permite e possibilita a interação com o usuário, de forma dinamizada, contribuindo para apropriação de conceitos matemáticos.

Neste contexto buscamos identificar que discussões tem sido privilegiadas nas investigações presentes em teses de doutorado e dissertações de mestrado no Catálogo online de Teses e Dissertações da CAPES, que tratam do software GeoGebra no ensino 
VIII Congresso Brasileiro de Informática na Educação (CBIE 2019)

Anais dos Workshops do VIII Congresso Brasileiro de Informática na Educação (WCBIE 2019)

da Matemática. Utilizamos como refinamento na busca, trabalhos com área de concentração em Educação Matemática, localizados por meio da palavra-chave GeoGebra dos quais na leitura dos títulos e resumos evidenciamos níveis de ensino envolvidos, os sujeitos da pesquisa, conteúdos matemáticos privilegiados e modalidade de investigação presentes nessas pesquisas.

Para tanto, apresentamos o software GeoGebra no contexto da Educação Matemática, algumas pesquisas brasileiras que relatam o uso potencial do software no Ensino da Matemática, a caracterização e discussão dos dados da pesquisa realizada. A última seção apresenta algumas considerações sobre o mapeamento realizado.

\section{O software GeoGebra no contexto da Educação Matemática}

Uma educação de qualidade para todos atualmente não pode ser obtida sem considerarmos a dimensão tecnológica [UNESCO 2016]. As novas tecnologias inegavelmente têm favorecido as possibilidades de experimentação visualização e simulação, no qual a discussão do uso pedagógico destes recursos nas aulas de Matemática tem sido foco de muitos estudos [Cyrino e Baldini 2012].

Assim, de acordo com esses estudos e mobilizados com o desafio em aliar a tecnologia ao ensino de Matemática pesquisadores tem discutido o uso de softwares como uma possibilidade, para realização de intervenções por meio de tarefas que favoreçam o aprendizado dos alunos. A utilização de softwares no ensino da Matemática para auxílio na resolução de tarefas tem se apresentado como uma alternativa que favorece a aprendizagem. Gafanhoto e Canavarro (2014) relatam que:

O uso de softwares no ensino, como os ambientes de geometria dinâmica (AGDs), desafia não só a abordagem matemática aos conhecimentos, mas também a dinâmica com que podem ser abordados, permitindo aos alunos uma grande autonomia na aprendizagem. (p. 115).

Os ambientes de geometria também auxiliam na resolução e no entendimento das tarefas, se bem utilizados pelos alunos. Para Canavarro e Gafanhoto (2014), citado em Gafanhoto (2011):

Entre estes destaca-se o GeoGebra, acessível de forma livre às escolas e aos alunos, com interface em português, e com potencialidades na múltipla representação de funções, incluindo a gráfica (representação gráfica de função em referencial cartesiano), a algébrica (escrita ou visualização da expressão algébrica da função), a tabular (mais ou menos completa e sobre domínio delimitado), e a numérica (coordenadas de pontos ou cálculo através da tabela) (p. 119).

Deste modo, este software tem se apresentado como uma ferramenta que permite a construção de pontos, vetores, segmentos, funções, cônicas e outros objetos matemáticos, favorecendo a investigação. As construções podem ocorrer de duas formas diferentes: utilizando as ferramentas disponíveis no software ou plotando, na caixa de entrada, as equações/coordenadas do objeto a ser criado. Outra característica que se destaca no GeoGebra é a possibilidade de manipular os objetos criados, o que atribui dinamismo às construções. Isto está relacionado ao controle deslizante, uma ferramenta que determina uma variável numérica, dentro de um intervalo pré- 
VIII Congresso Brasileiro de Informática na Educação (CBIE 2019)

Anais dos Workshops do VIII Congresso Brasileiro de Informática na Educação (WCBIE 2019)

estabelecido, que varia de acordo com um incremento, ações determinadas pelo operador. Assim, no caso em que um objeto depende de um controle deslizante, quando o usuário altera seu valor, ou utiliza a ferramenta animar, confere dinamicidade à construção [Basniak 2018].

$\mathrm{Na}$ seção seguinte destacamos alguns dos trabalhos que vem enfatizando as potencialidades no processo de ensino da Matemática com o software GeoGebra no contexto da Educação Matemática.

\section{Contexto das pesquisas brasileiras e o uso do software GeoGebra no Ensino da Matemática}

Cyrino e Baldini (2012), realizaram um estudo investigativo de teses e dissertações disponíveis no Banco de dados da CAPES, com foco na formação de professores de Matemática, na busca de compreender quais discussões estavam sendo privilegiadas e conhecer as perspectivas presentes nestas pesquisas. Para seleção dos trabalhos a serem analisados as pesquisadoras utilizaram "GeoGebra" como palavra-chave, encontrando uma tese de Doutorado, 8 dissertações de Mestrado Acadêmico e 27 dissertações de Mestrado Profissional, publicadas no período de 2008 a 2010. A partir da leitura dos resumos dos trabalhos, verificaram que um deles não utilizou o GeoGebra e foi desconsiderado, totalizando 35 trabalhos. Em seguida, realizaram uma análise interpretativa do resumo, da introdução, do encaminhamento metodológico e das considerações finais desses trabalhos.

Amaral e Frango (2014) realizaram um levantamento de teses e dissertações, que tratavam do uso do software GeoGebra no ensino de funções matemáticas no período de 2008 a 2012, com o intuito de validar o tema "Contribuições do uso de Tecnologias de Informação e Comunicação (TICs) na aprendizagem de funções matemáticas, no Brasil". Os autores, realizaram buscas, on-line, em publicações no banco de teses da Coordenação de Aperfeiçoamento de Pessoal de Nível Superior (CAPES), no portal de periódicos também da CAPES, no site da Biblioteca Digital de Teses e Dissertações (BDTD) e no site Domínio Público. Como chaves de pesquisa foram definidas as palavras "ensino de funções", "ensino médio brasileiro" e "GeoGebra". Foram encontradas apenas dissertações, totalizando 35, e essas foram categorizadas de acordo com os seus objetivos e os procedimentos metodológicos utilizados. Para o mapeamento proposto, foram utilizados pelos autores como material de análise o total de 20 publicações.

Lemke, Silveira e Siple (2016) realizaram uma investigação de teses e dissertações com o objetivo de verificar as pesquisas que contemplaram a tecnologia GeoGebra no ensino brasileiro, no período de 2013 a maio de 2016. Para realizar essa investigação optaram, por uma análise preliminar, procurando pelos trabalhos que contemplassem o GeoGebra como palavra-chave, sendo selecionados o site da Biblioteca Digital de Teses e Dissertações (BDTD), o site Domínio Público e as dissertações do Mestrado Profissional em Matemática em Rede Nacional (PROFMAT). Foram realizadas na investigação uma leitura preliminar das teses e dissertações, contemplando os títulos dos trabalhos, a fim de verificarem se um mesmo trabalho estava disponível em mais de um desses bancos de dados. Ao mesmo tempo observaram as palavras-chave, para ver se realmente o uso do GeoGebra se fazia presente no 
VIII Congresso Brasileiro de Informática na Educação (CBIE 2019)

Anais dos Workshops do VIII Congresso Brasileiro de Informática na Educação (WCBIE 2019)

desenvolvimento do trabalho. Os autores analisaram 308 teses e dissertações, sendo 9 teses e 299 dissertações, das quais 8 de Mestrado Acadêmico e 291 de Mestrado Profissional, sendo 272 do PROFMAT.

Diante do estudo dos trabalhos citados anteriormente e com o intuito de contribuirmos com um levantamento atual referente ao tema, qual seja, o software GeoGebra no ensino da Matemática, realizamos uma pesquisa investigativa de teses de doutorado e dissertações de mestrados brasileiros, sendo caracterizada e realizada uma discussão acerca dos dados coletados na seção seguinte.

\section{Caracterização e discussão dos dados da Pesquisa}

No segundo semestre de 2019, recorremos ao Catálogo online de Teses e Dissertações da CAPES, com o objetivo de identificar quais discussões tem sido privilegiadas nas investigações envolvendo o software GeoGebra no Ensino de Matemática presentes em teses de doutorado e dissertações de mestrado acerca do uso do software GeoGebra no Ensino da Matemática. Utilizamos para busca dos trabalhos a serem analisados GeoGebra como palavra-chave. Encontramos num primeiro momento 1039 trabalhos entre teses e dissertações e optamos por analisar apenas as publicações entre 2017 e 2018. Com esse refinamento, na busca apareceram 159 publicações em 2017 e 175 em 2018, totalizando 334 trabalhos. Com objetivo de analisarmos apenas os trabalhos que tratassem do uso do GeoGebra, em programas de doutorado e mestrado com a área de concentração e ênfase em Educação Matemática, optamos por esse refinamento e encontramos nessa busca 25 publicações, sendo 07 teses de doutorado, 12 de Mestrado Acadêmico e 6 de Mestrado Profissional, as quais foram objeto da nossa investigação.

Para a análise dos 25 trabalhos selecionados, relacionados a seguir destacamos respectivamente, autor em ordem alfabética, título, ano de defesa e instituição. Na investigação propomos um código para cada trabalho, adaptando a proposta utilizada por Cyrino e Baldini (2012), criando um código composto por uma sigla e um número. A sigla indica a modalidade, quais sejam, (T) para tese de doutorado, (DMA) para Dissertação de Mestrado Acadêmico e (DMP) para Mestrado Profissional, e o número representa a ordem em que o trabalho foi analisado. Por exemplo, DMA2 representa, dentre os trabalhos selecionados, uma "dissertação de mestrado acadêmico" que foi o segundo trabalho analisado. Este código não tem a função de ordenar os trabalhos a partir de um critério específico, ele foi utilizado para associar, posteriormente, cada trabalho com a(s) sua(s) respectiva(s) palavras-chave e modalidades de investigação.

No quadro 1 apresentamos a relação dos trabalhos por autor e código, título, ano de publicação, e instituição de realização:

Quadro 1: Autor e código, título, ano de publicação e instituição.

\begin{tabular}{|c|c|c|c|}
\hline Autor e código & \multicolumn{1}{|c|}{ Título } & Ano & Instituição \\
\hline $\begin{array}{c}\text { Alan Silva dos Santos } \\
\text { (DMA18) }\end{array}$ & $\begin{array}{l}\text { Um estudo sobre o conceito de densidade do } \\
\text { conjunto dos números racionais e do conjunto } \\
\text { dos números irracionais: uma abordagem com } \\
\text { tecnologias. }\end{array}$ & 2017 & PUC-SP \\
\hline
\end{tabular}


VIII Congresso Brasileiro de Informática na Educação (CBIE 2019)

Anais dos Workshops do VIII Congresso Brasileiro de Informática na Educação (WCBIE 2019)

\begin{tabular}{|c|c|c|c|}
\hline $\begin{array}{l}\text { Andressa Maria da Cruz } \\
\text { (DMP8). }\end{array}$ & $\begin{array}{l}\text { Potencialidades da utilização do software } \\
\text { GeoGebra para o desenvolvimento do } \\
\text { conteúdo de funções exponenciais através do } \\
\text { smartphone. }\end{array}$ & 2018 & UFOP \\
\hline $\begin{array}{c}\text { Erica Maria Renno } \\
\text { Villela Dario (DMA14) }\end{array}$ & $\begin{array}{l}\text { Produtos notáveis no } 8^{\circ} \text { ano do Ensino } \\
\text { Fundamental II: contribuições da utilização de } \\
\text { diferentes recursos didáticos. }\end{array}$ & 2017 & PUC-SP \\
\hline $\begin{array}{l}\text { Gina Magali Horvath } \\
\text { Miranda (T22) }\end{array}$ & $\begin{array}{l}\text { Esculturas matemáticas: a integral dupla em } \\
\text { 3D. }\end{array}$ & 2018 & UNIAN \\
\hline $\begin{array}{l}\text { Gioconda Guadalupe } \\
\text { Cristales Flores (DMA3) }\end{array}$ & $\begin{array}{l}\text { A construção de mosaicos no plano por um } \\
\text { aluno com transtorno do espectro autista. }\end{array}$ & 2018 & UFSM \\
\hline $\begin{array}{l}\text { Hakel Fernandes de } \\
\text { Awila (DMA12) }\end{array}$ & $\begin{array}{l}\text { Uma análise da contribuição do GeoGebra } \\
\text { como recurso interativo para o estudo de áreas } \\
\text { e volumes. }\end{array}$ & 2017 & UFSM \\
\hline $\begin{array}{l}\text { Hebber Kennady Martins } \\
\text { dos Santos (DMP10) }\end{array}$ & $\begin{array}{l}\text { Ponto, reta e plano em ambientes lápis e papel } \\
\text { e GeoGebra: conhecimento dos alunos no } 6^{\circ} \\
\text { ano do Ensino Fundamental no município de } \\
\text { Paraupebas - Pará. }\end{array}$ & 2017 & UEPB \\
\hline $\begin{array}{l}\text { Hercules Nascimento } \\
\text { Silva (DMA6) }\end{array}$ & $\begin{array}{l}\text { Estudo de função: uma proposta de } \\
\text { reconstrução de atividades do imagiciel } \\
\text { mediadas pelo GeoGebra. }\end{array}$ & 2017 & PUC-SP \\
\hline $\begin{array}{l}\text { Jeferson da Silva } \\
\text { Gonçalves (T21) }\end{array}$ & $\begin{array}{l}\text { Relações entre funções inversa e composta: } \\
\text { uma proposta de exploração dos conceitos } \\
\text { com auxílio do software GeoGebra }\end{array}$ & 2017 & UNIAN \\
\hline $\begin{array}{l}\text { Joel Silva de Oliveira } \\
\text { (DMA5) }\end{array}$ & $\begin{array}{l}\text { A Engenharia Didática como Referencial para } \\
\text { a Ação Pedagógica Reflexiva: O Caso da } \\
\text { Área de Figuras Planas Irregulares com o } \\
\text { GeoGebra. }\end{array}$ & 2017 & UEPB \\
\hline $\begin{array}{l}\text { Joice Yuko Obata } \\
\text { (DMA16) }\end{array}$ & $\begin{array}{l}\text { As tic no ensino de Matemática: o que as } \\
\text { produções didático-pedagógicas do PDE nos } \\
\text { dizem? }\end{array}$ & 2018 & UFPR \\
\hline $\begin{array}{l}\text { Jose Ronaldo Alves } \\
\text { Araujo (DMA9) }\end{array}$ & $\begin{array}{l}\text { Atividades para o estudo das medidas de } \\
\text { tendência central: uma proposta com o apoio } \\
\text { do GeoGebra. }\end{array}$ & 2018 & PUC-SP \\
\hline $\begin{array}{l}\text { Josias Julio de Araujo } \\
\text { (DMP1) }\end{array}$ & $\begin{array}{l}\text { O software GeoGebra numa proposta de } \\
\text { formação continuada de professores de } \\
\text { Matemática do Ensino Fundamental. }\end{array}$ & 2017 & UFOP \\
\hline $\begin{array}{l}\text { Juliane Carla Berlanda } \\
\text { (DMA7) }\end{array}$ & $\begin{array}{l}\text { Mobilizações de registros de representação } \\
\text { semiótica no estudo de trigonometria no } \\
\text { triângulo retângulo com o auxílio do software } \\
\text { GeoGebra. }\end{array}$ & 2017 & UFSM \\
\hline $\begin{array}{c}\text { Kesia de Melo } \\
\text { Hermenegildo (DMP2) }\end{array}$ & $\begin{array}{l}\text { Os saberes da formação inicial do professor } \\
\text { para a integração da investigação em } \\
\begin{array}{l}\text { Matemática com recursos da geometria } \\
\text { dinâmica. }\end{array}\end{array}$ & 2017 & UEPB \\
\hline
\end{tabular}


VIII Congresso Brasileiro de Informática na Educação (CBIE 2019)

Anais dos Workshops do VIII Congresso Brasileiro de Informática na Educação (WCBIE 2019)

\begin{tabular}{|c|c|c|c|}
\hline $\begin{array}{l}\text { Luciano Augusto da } \\
\text { Silva Melo (T25) }\end{array}$ & $\begin{array}{l}\text { Tradução interna e Jogos de imagens na } \\
\text { Matemática }\end{array}$ & 2018 & UFPA \\
\hline $\begin{array}{l}\text { Marcio Antonio Cometti } \\
\text { (DMP4) }\end{array}$ & $\begin{array}{l}\text { Discutindo o ensino de integrais múltiplas no } \\
\text { cálculo de várias variáveis: contribuições do } \\
\text { GeoGebra 3D para a aprendizagem. }\end{array}$ & 2018 & UFOP \\
\hline $\begin{array}{l}\text { Marcio Vieira de } \\
\text { Almeida (T24) }\end{array}$ & $\begin{array}{l}\text { Material para o Ensino do Cálculo Diferencial } \\
\text { e Integral: referências de TALL, Gueudet e } \\
\text { Trouche }\end{array}$ & 2017 & PUC-SP \\
\hline $\begin{array}{l}\text { Maria Rosana Soares } \\
\text { (T23) }\end{array}$ & $\begin{array}{l}\text { Um estudo da arte das pesquisas acadêmicas } \\
\text { sobre modelagem em Educação Matemática } \\
(1979 \text { a 2015) nas áreas de Educação e } \\
\text { Ensino da Capes: as dimensões } \\
\text { fundamentadas e as direções históricas. }\end{array}$ & 2017 & PUC-SP \\
\hline $\begin{array}{l}\text { Pedro Henrique da Silva } \\
\text { (DMP13) }\end{array}$ & $\begin{array}{l}\text { Transformações geométricas no contexto } \\
\text { escolar: uma experiência de aprendizagem no } \\
8^{\circ} \text { ano do Ensino Fundamental. }\end{array}$ & 2017 & UFOP \\
\hline $\begin{array}{l}\text { Priscila Arcego } \\
\text { (DMA17) }\end{array}$ & $\begin{array}{l}\text { Representações semióticas mobilizadas no } \\
\text { estudo da área do círculo no Ensino } \\
\text { Fundamental. }\end{array}$ & 2017 & UFSM \\
\hline $\begin{array}{l}\text { Roberto Seidi Imafuku } \\
\text { (T19) }\end{array}$ & $\begin{array}{l}\text { O uso dos softwares GeoGebra e SimCalc } \\
\text { para enriquecimento da imagem de conceito } \\
\text { de derivada. }\end{array}$ & 2018 & UNIAN \\
\hline $\begin{array}{l}\text { Rubervan da Silva Leite } \\
\text { (DMA15) }\end{array}$ & $\begin{array}{l}\text { Formação de professores de Matemática e } \\
\text { tecnologias digitais: um estudo sobre o } \\
\text { Teorema de Tales. }\end{array}$ & 2017 & PUC-SP \\
\hline $\begin{array}{l}\text { Wendel de Oliveira Silva } \\
\text { (T20) }\end{array}$ & $\begin{array}{l}\text { Formação continuada: um estudo sobre } \\
\text { integração de tecnologia digital para ensinar } \\
\text { poliedros. }\end{array}$ & 2018 & UNIAN \\
\hline $\begin{array}{l}\text { Willians Adriano de } \\
\text { Oliveira (DMA11) }\end{array}$ & $\begin{array}{l}\text { Tecnologias Digitais na } \\
\text { continuada: situações de Ensino Articulando } \\
\text { Geometria e Funções. }\end{array}$ & 2017 & UNIAN \\
\hline
\end{tabular}

Com esse primeiro análise constatamos que onze trabalhos apresentaram a palavra GeoGebra no título, sete no ano de 2017 e quatro em 2018. Observamos também que as instituições de defesa dos trabalhos estão localizadas em sua maioria na região Sudeste com dezesseis trabalhos, cinco na região Sul e quatro no Nordeste.

Em seguida realizamos uma análise interpretativa dos resumos desses trabalhos e identificamos os sujeitos da pesquisa (Quadro 2) e os conteúdos matemáticos abordados (Quadro 3). A partir dessa leitura em três trabalhos não conseguimos identificar os sujeitos da pesquisa, e também em outro não identificamos o conteúdo matemático abordado por se tratar de uma pesquisa de produção didático pedagógica no âmbito da formação de professores.

Quadro 2: Sujeitos da Pesquisa 
VIII Congresso Brasileiro de Informática na Educação (CBIE 2019)

Anais dos Workshops do VIII Congresso Brasileiro de Informática na Educação (WCBIE 2019)

\begin{tabular}{|l|c|}
\hline \multicolumn{1}{|c|}{ Sujeitos da Pesquisa } & Quantidade \\
\hline Estudantes do Ensino Fundamental & 05 \\
\hline Estudantes do Ensino Médio & 05 \\
\hline Estudantes de Licenciatura em Matemática & 06 \\
\hline Estudantes de Engenharia & 01 \\
\hline Professores da Educação Básica & 05 \\
\hline Não Informado no Resumo & 03 \\
\hline
\end{tabular}

Quadro 3: Conteúdos matemáticos contemplados

\begin{tabular}{|l|c|}
\hline \multicolumn{1}{|c|}{ Conteúdos de Matemática } & Quantidade \\
\hline Funções & 05 \\
\hline Geometria Plana & 07 \\
\hline Álgebra e Geometria & 02 \\
\hline Teorema de Pitágoras & 01 \\
\hline Estatística & 01 \\
\hline Trigonometria no triângulo retângulo & 01 \\
\hline Cálculo & 05 \\
\hline Geometria espacial/plana & 02 \\
\hline Teorema de Talles & 01 \\
\hline Produtos notáveis & 01 \\
\hline Não informado & 01 \\
\hline
\end{tabular}

A seguir elencamos de cada trabalho a(s) sua(s) respectiva(s) palavras-chave e modalidades de investigação de acordo com a ordem das leituras e com os códigos do quadro 1:

DMP1: Palavras-chave: Formação Continuada; Ensino de Álgebra e Geometria; GeoGebra. Modalidade de Investigação: Pesquisa Qualitativa; Pesquisa teórico bibliográfica e Pesquisa de Campo.

DMP2: Palavras-chave: Investigação Matemática; TIC; GeoGebra; Formação de Professores Modalidade de Investigação: Pesquisa Qualitativa; pesquisa pedagógica.

DMA3: Palavras-chave: Transtorno do Espectro Autista; Teoria da Aprendizagem Significativa; GeoGebra; mosaicos. Modalidade de Investigação: Pesquisa qualitativa; estudo de caso; teoria da aprendizagem significativa.

DMP4: Palavras-chave: GeoGebra 3D; Ensino de Cálculo de Várias Variáveis; Ensino de Integrais Múltiplas; Teoria dos Registros das Representações Semióticas; Educação Matemática no Ensino Superior. Modalidade de Investigação: Pesquisa qualitativa; 
VIII Congresso Brasileiro de Informática na Educação (CBIE 2019)

Anais dos Workshops do VIII Congresso Brasileiro de Informática na Educação (WCBIE 2019)

Teoria dos Registros das representações semióticas, sequência didática.

DMA5: Palavras-chave: Engenharia Didática; figuras planas irregulares; GeoGebra. Modalidade de Investigação: Pesquisa qualitativa; pesquisa intervenção; engenharia didática.

DMA6: Palavras-chave: Imagiciel; GeoGebra; Função real definida por sentenças; aprendizagem. Modalidade de Investigação: Pesquisa qualitativa; Engenharia Didática.

DMA7: Palavras-chave: Trigonometria no triângulo retângulo; GeoGebra; Registros de representação semiótica; Engenharia didática. Modalidade de Investigação: Pesquisa qualitativa; Registro de representação semiótica e engenharia didática.

DMP8: Palavras-chave: Dispositivos Móveis; Funções Exponenciais; Smartphone; Software GeoGebra; Tecnologias Digitais; Teoria Fundamentada nos Dados. Modalidade de Investigação: Pesquisa Qualitativa; teoria fundamentada.

DMA9: Palavras-chave: GeoGebra (Software); Medidas de Tendência Central; Teoria das Situações Didáticas; Engenharia didática. Modalidade de Investigação: Pesquisa qualitativa; Teoria das situações didáticas; Engenharia didática.

DMP10: Palavras-chave: Educação Matemática; GeoGebra; Ensino Fundamental; Geometria Plana. Modalidade de Investigação: Pesquisa qualitativa; observação participante.

DMA11: Palavras-chave: Educação Matemática; Educação Continuada; TPACK; GeoGebra; Áreas; Funções Quadráticas. Modalidade de Investigação: Pesquisa Qualitativa; pesquisa documental.

DMA12: Palavras-chave: Área; Volume; Área e volume; Sequência didática; GeoGebra. Modalidade de Investigação: Pesquisa qualitativa; pesquisa de campo; pesquisa-ação.

DMP13: Palavras-chave: transformações geométricas; ensino-aprendizagem; Ensino Fundamental. Modalidade de Investigação: Pesquisa qualitativa; pesquisa documental.

DMA14: Palavras-chave: Produtos notáveis; Matemática (Ensino Fundamental); Educação de base - Brasil. Modalidade de Investigação: Pesquisa qualitativa; pesquisa intervenção.

DMA15: Palavras-chave: Professores de Matemática - Formação profissional; Educação Matemática - Inovações tecnológicas; Conhecimento Tecnológico e Pedagógico do Conteúdo (TPACK). Modalidade de Investigação: Pesquisa qualitativa;

DMA16: Palavras-chave: TIC; PDE; Formação de professores; Hermenêutica. Modalidade de Investigação: Pesquisa qualitativa; fenomenologia.

DMA17: Palavras-chave: Registros de representação semiótica; Apreensões; Ensino de geometria; Área do círculo; Ensino Fundamental. Modalidade de Investigação: Pesquisa qualitativa; registros de representação semiótica.

DMA18: Palavras-chave: Educação Matemática; Matemática - Estudo e ensino; Números racionais. Modalidade de Investigação: Pesquisa qualitativa; teoria das situações didáticas. 
T19: Palavras-chave: SimCalc; GeoGebra; Concepções de Derivada; Três mundos da Matemática; Imagem de Conceito. Modalidade de Investigação: Pesquisa Qualitativa; Teoria dos três mundos da Matemática (TALL).

T20: Palavras-chave: Educação Matemática; Aulas investigativas; Formação continuada em Matemática; TPACK; GeoGebra 3D. Modalidade de Investigação: Pesquisa Qualitativa; Design-Based Research

T21: Palavras-chave: Educação Matemática; Funções Composta e Inversa; Três mundos da Matemática; Registros de Representação Semiótica; Design Experiment. Modalidade de Investigação: Pesquisa Qualitativa; Registros de Representação Semiótica, Teoria dos três mundos da Matemática (TALL), Design experiment.

T22: Palavras-chave: Função de duas variáveis; Integral Dupla; Tecnologia; Impressão 3D; Teoria da cognição corporificada. Modalidade de Investigação: Pesquisa Qualitativa; Design experiment.

T23: Palavras-chave: Cálculo Diferencial-Estudo e Ensino; Cálculo Integral-Estudo e Ensino; Educação Matemática-Ensino com auxílio do computador. Modalidade de Investigação: Pesquisa Qualitativa; descritiva.

T24: Palavras-chave: Cálculo Diferencial-Estudo e Ensino; Cálculo Integral-Estudo e Ensino; Educação Matemática-Ensino com auxílio do computador. Modalidade de Investigação: Pesquisa Qualitativa; fundamental.

T25: Palavras-chave: Educação Matemática; Wittgenstein; Jogos de linguagem; tradução interna; Jogos de imagens na Matemática. Modalidade de Investigação: Pesquisa Qualitativa.

Nas leituras realizadas no trabalho DMP1 não conseguimos identificar a modalidade de investigação, sendo necessário a leitura da seção metodológica da dissertação. Nos trabalhos DMP13, DMA14, DMA15, DMA16, DMA17, DMA18, T21, T22, T23, T24, T25, o GeoGebra não foi mencionado como palavra-chave. Todos os trabalhos se utilizaram do método e estratégias qualitativas de investigação, o que de acordo com Creswell (2010 p. 211) "é uma pesquisa interpretativa, com o investigador tipicamente envolvido em uma experiência sustentada e intensiva com os participantes".

Ainda, destacamos as escolhas dos trabalhos DMP4, DMA5, DMA6, DMA7, DMA9, DMA17, DMA18, T21, pelas teorias da Didática da Matemática, modelos teóricos da escola francesa, que segundo Almoloud (2007 p. 17) "é vista como uma ciência que tem por objetivo investigar os fatores que influenciam o ensino $\mathrm{e}$ aprendizagem de Matemática e o estudo de condições que favorecem a sua aquisição pelos alunos".

\section{Algumas Considerações}

O que nos mobilizou nesse estudo foi identificar que discussões têm sido privilegiadas nas investigações envolvendo o software GeoGebra no Ensino da Matemática, buscando no banco de teses online da CAPES teses de doutorado e dissertações de mestrados que apresentassem a palavra-chave GeoGebra, em programas de Pós graduação com área de concentração em Educação Matemática, realizando assim uma análise investigativa 
interpretativa, por meio da leitura dos títulos e dos resumos dos trabalhos, após a qual, mapeamos os sujeitos da pesquisa, níveis de ensino envolvidos, os conteúdos matemáticos privilegiados, palavras-chave e modalidade de investigação.

Em alguns dos trabalhos analisados observamos nos sujeitos da pesquisa a preocupação da maioria dos pesquisadores, em discutir as potencialidades do software GeoGebra em aulas de matemática no que se refere a formação de professores, tanto na licenciatura, quanto para professores em atuação na Educação Básica. Ainda nas dissertações, os conteúdos matemáticos em sua maioria abordados se referem a geometrias, sugerindo que as potencialidades que o software oferece ainda poderão em novas pesquisas serem mobilizadas e amplamente exploradas. Em relação a modalidade de investigação das pesquisas todas se utilizaram do método e estratégias qualitativas de investigação.

Com a investigação realizada percebemos a importância do software GeoGebra no âmbito da Educação Matemática, como mediador da aprendizagem, e a relevância das pesquisas brasileiras que tem debatido as potencialidades e possibilidades para o Ensino da Matemática buscando assim contribuir para novos estudos e enfoques dessa tecnologia.

A continuidade de pesquisas nessa temática podem auxiliar no esclarecimento de dificuldades para o uso potencial do software em conteúdos matemáticos e formas de abordagem. Sugerimos investigar trabalhos futuros que tratem de simulações e animações utilizando o GeoGebra, e os aspectos potenciais desencadeados pelos sujeitos envolvidos, para aprendizagem de conceitos matemáticos.

\section{Referências}

Almoloud, S. Ag. (2007), Fundamentos da Didática da Matemática. Curitiba: Editora UFPR. 218p.

Amaral, M.P., Frango, I. (2014) "Um levantamento sobre pesquisas com o uso do software GeoGebra no ensino de funções matemáticas", In: Revista Eletrônica de Educação Matemática - Revemat. Florianópolis (SC), v.9, n. 1, p. 90-107.

Basniak, M. I. (2019) “A construção de animações e simuladores no software GeoGebra e o Ensino e a Aprendizagem de Matemática", Projeto de pesquisa MCTIC/CNPq 2018. Disponível em <cnpq.br/projetos-pesquisa>. Acesso em: 24 de abr.

Creswell, J. W. (2010), Projeto de pesquisa: métodos qualitativo, quantitativo e misto. Tradução Magda Lopes. 3. ed. rev. Porto Alegre: Artmed.

Cyrino, M. C. C. T., \& Baldini, L. A. F. (2012) "O software GeoGebra na formação de professores de matemática - uma visão a partir de dissertações e teses", Revista Paranaense de Educação Matemática, v.1, n.1, p. 42-61.

Estevam, E., Basniak, M. I., Paulek, C. M., Scaldelai, D., Felipe, N. (2018) "Ensino Exploratório de Matemática e Tecnologias Digitais: a elaboração da lei dos senos mediada pelo software GeoGebra”, Acta Scientiae, Canoas, v. 20, n. 3, p. 342-358.

Gafanhoto, A. P., Canavarro, A. P. (2014), A adaptação das tarefas matemáticas: Como promover o uso de múltiplas representações. In: PONTE, J. P. (Org.). Práticas Profissionais dos Professores de Matemática (p. 113-134). Lisboa: IEUL. 
VIII Congresso Brasileiro de Informática na Educação (CBIE 2019)

Anais dos Workshops do VIII Congresso Brasileiro de Informática na Educação (WCBIE 2019)

LEMKE, R., SILVEIRA, R. F., \& SIPLE, I. Z. GeoGebra: uma tendência no ensino de Matemática. Anais do Colóquio Brasileiro de Educação, 2, Joinville, Brasil, p. 607 619. 2016.

UNESCO. Os desafios do ensino da matemática na Educação Básica. Brasília. São Carlos: EdUFSCar: 2016. 114p. 